\title{
A Simulated Discrete-Event and Queuing Model to Reduce Transfers from the Emergency Department and to Optimize Hospital Bed Management
}

\author{
M. Wargon, ${ }^{1,2,3}$ N. Taright, ${ }^{4}$ E. Casalino, ${ }^{3,5,6}$ D. Pateron, ${ }^{1,4}$ and B. Guidet ${ }^{1,7}$ \\ ${ }^{1}$ Université Pierre et Marie Curie-Paris 6, UMR S 707, 75012 Paris, France \\ ${ }^{2}$ Hôpital Saint Camille, 94360 Bry-sur-Marne, France \\ ${ }^{3}$ Study Group on Efficiency and Quality in Non-Scheduled Activities, 75018 Paris, France \\ ${ }^{4}$ Department of Medical Information, AP-HP, Hôpital Saint Antoine, 75012 Paris, France \\ ${ }^{5}$ Emergency Department, AP-HP, Hôpital Bichat-Claude Bernard, 75018 Paris, France \\ ${ }^{6}$ Université Paris Diderot, Sorbonne Paris Cité, UA REMES, 75013 Paris, France \\ ${ }^{7}$ Department of Intensive Care, AP-HP, Hôpital Saint Antoine, 75012 Paris, France \\ Correspondence should be addressed to M. Wargon; m.wargon@ch-bry.org
}

Received 18 August 2014; Accepted 9 November 2014; Published 1 December 2014

Academic Editor: Michael Blaivas

Copyright (C) 2014 M. Wargon et al. This is an open access article distributed under the Creative Commons Attribution License, which permits unrestricted use, distribution, and reproduction in any medium, provided the original work is properly cited.

\begin{abstract}
Objectives. Emergency departments (EDs) and elective hospitalizations compete for beds. Our aim was to reduce hospital transfers using a queuing-model study. Methods. Macros were created to simulate four priority groups of patients according to hospitalization mode (elective, ED) and age ( $\geq 75$ and $<75$ years), with randomization of number of admissions and length of stay (LOS). Those priorities were assigned regarding usual situations (ED admission with less priority than scheduled admission) not regarding clinical contexts. Simulations were based on actual data from an academic hospital. Models simulated ED boarder queue according to different scenarios based on number of hospital beds, LOS, and preventable hospitalizations. Results. Observed hospital-LOS was longer for patients $\geq 75$ years $(12.2 \pm 3.6$ days versus $11.4 \pm 3.8$ days; $P<0.01)$ and for ED admissions $(12.2 \pm 0.6$ versus $9.7 \pm 0.6$ days; $P<0.01$ ). In simulation models, two scenarios stabilized the beds demand after admissions: limitation of LOS to 30 days or $20 \%$ decrease in elective admissions among older patients. With these scenarios, the queue would be 25.2 patients for 361 beds $(+2 \%)$ and 16.7 patients for 354 beds. Conclusions. Queuing models offer an interesting approach to bed management. A significant reduction in ED transfers is feasible, by limiting LOS to $<30$ days or by reducing elective hospitalizations of patients by $20 \%$.
\end{abstract}

\section{Introduction}

Access block is one of the principal factors influencing ED overcrowding $[1,2]$. Access blocking and "boarding" have been previously associated with an increased risk of errors, delayed time to critical-care admission, and increased morbidity and mortality [1]. Hospitalizations through EDs (unplanned or nonelective) compete with elective admissions (planned or direct) for scarce medical and surgical ward beds [3]. Unplanned patients are the most affected because they frequently have acute medical conditions with older patients having an increased risk [4].
Factors influencing the availability of beds for nonelective hospitalizations and the rates of hospital bed occupancy have been outlined previously [5] and these factors extend the amount of time spent in the ED [6]. In order to forecast ED activity [7], a global hospital approach is required and the need for beds should be assessed on a daily basis [8]. There is great variability in admissions, type of patients, and distribution of hospital length of stay (LOS) depending on the day of admission [9] suggesting that computer simulation is the best tool to test different scenarios. Regarding the great variability in ED visits [10], expanding the size of EDs has no impact 
on reducing ED-LOS whereas improving the rate of hospital admissions is effective [5].

In the areas of Paris it is estimated that up to $2.5 \%$ of ED visits and $8-25 \%$ of patients admitted in ED are transferred to other institutions. In the US, $1.9 \%$ of ED visits result in transfers to other hospitals [11]. Transfers or diversions have been associated with increased costs, income loss, and reduced quality of care [12]. Although interfacility transfer is considered to be an integral part of any health-care system, the reduction in number of transfers is a priority in some time-critical conditions [13].

It is currently accepted that improving ED quality includes discrete-event simulation and queuing systems [14]. Most simulations focus on EDs or on bed needs from an ED perspective $[5,15]$. We hypothesized that hospitals have the potential to admit more unplanned patients.

The primary objective was to evaluate the effects of different hospital bed management strategies including hospital bed number, number of boarding patients, and in-hospitalLOS.

\section{Methods}

2.1. Study Design. We constructed an Excel sheet with macros in VBA (Excel 2002) to simulate different types of admissions in a virtual hospital.

2.2. Selection of Data. Data from a French academic hospital were used for the simulations. The hospital has 354 medical beds and its ED receives approximately 50,000 patients each year. The ED observation unit (OU) has 22 beds. Data collected included admission date, type of admission (via the ED or elective to medical wards), age category ( $\geq 75$ and $<75$ years), and day of the week (Monday to Sunday). In our facilities, problems occur more often with medical patients than surgical patients and our organization separates day procedure from complete hospitalization. Day-cases units do not receive patients for full hospitalization and life threatening patients are not kept in boarding but transferred immediately. For these reasons, only beds from medical departments were included, except for day-cases and intensive care units.

2.3. Ethical Approval. The dataset is currently used as an ED and hospital quality and performance measure as part of an ongoing activity and performance evaluation approved by the Assistance Publique-Hôpitaux de Paris committees on research and informatics. The databases are registered with the French Data Protection Authority (CNIL) for medical and research purposes. Given its design, aggregating data analysis with no individual identifiers, this study did not require individual informed consent.

2.4. Model. The model generates different types of admissions every day of a 365-day year for 1000 simulations. The program deals with three types of admission: (i) priority, (ii) nonpriority, and (iii) admitted patients already boarding in the ED (queue). For each priority group, two subtypes were considered: $\geq 75$ years of age and $<75$ years. By convention, elective admissions were categorized as priority while admissions through the ED were classified as nonpriority, ED boarders being admitted before new ED patients.

Each day, the simulation generates a number of "patients" randomly according to a normal distribution. The actual average daily admissions were determined by day of the week for each subtype. For each "patient," a LOS was randomly chosen from the distribution probability of hospital-LOS for the subtype according to the day of admission. Actual hospitalLOS for the different subtypes was used in the base scenario. Then virtual patients were admitted according to their order of priority (first, elective patients and then ED boarders and finally newly generated ED patients, as long as vacant beds were available (Figure 1)). If there were no more vacant beds, it was decided that priority patients should be accommodated by other means, and nonpriority patients were added to the queue of ED boarders. LOS for admitted patients was decremented each day before the admissions process. When a patient reached a LOS of 0 , the bed was vacant. As long as a patient was boarding in the ED, LOS was not decremented.

We defined 22 patients per night (ppn) as a queuing value indicating ED boarding capacity guaranteeing quality of care, not exceeding the existing ED-OU number of beds. The EDOU plays an important role in the reception of older patients [16].

2.5. Study Groups. Four groups were constructed: (i) priority group (PG) 1: elective hospitalizations for patients aged $\geq 75$ years; (ii) PG 2: elective hospitalizations for patients aged $<75$ years; (iii) non-PG 1: emergency hospitalizations for patients aged $\geq 75$ years; and (iv) non-PG 2: emergency hospitalizations for patients aged $<75$ years.

2.6. Outputs. We chose to use the last 90 days of the simulation after a stabilisation of the simulation. The main measurement of the performance was the number of patients boarding in the ED. In our model, patients boarding in the $\mathrm{ED}$ on stretchers or in a bed in the OU were not distinguished as both were waiting for a hospital admission in the ED.

2.7. Study Hypothesis. We constructed different hypotheses. For each scenario, the number of hospital beds was increased by increments of $2 \%$ up to $10 \%$. Evolution of ED boarding queue was computed.

(i) Hypothesis 1 (HYP1): a sensitivity analysis was performed to calculate the number of beds necessary to accommodate all ED patients, including transfers. However, we considered that $15 \%$ of younger patients and $5 \%$ of elderly patients who were transferred had to be transferred anyway (no specialty in the hospital, already treated in another institution, etc.). This first assumption was maintained in the following scenarios.

(ii) Hypothesis 2 (HYP-M-LOS): it was decided to keep the same LOS probability distribution identical to a Monday distribution for each subtype, Mondays LOS being the most efficient in the actual series. 


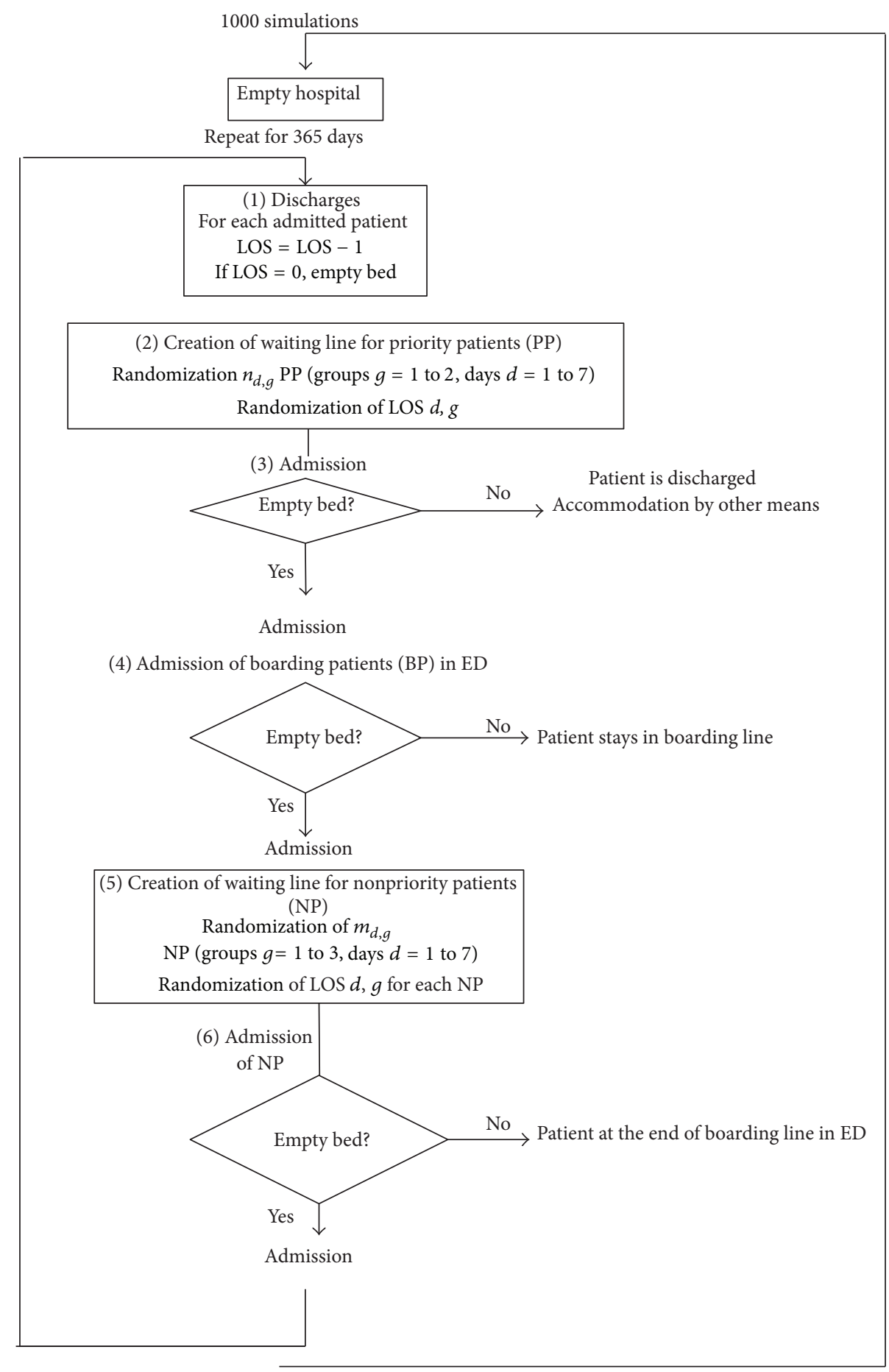

FIGURE 1: Simulation process.

(iii) Hypothesis 3 (HYP-30D): in this scenario, it was decided to simulate an original LOS distribution terminated at 30 days.

(iv) Hypothesis 4 (HYP-MtoTWT): as Monday is the most crowded day for planned admissions, $40 \%$ of hospitalizations in priority subgroup 2 (planned hospitalizations among patients $<75$ years) were diverted from Monday to Tuesday (20\%), Wednesday (10\%), or Thursday (10\%).

(v) Hypothesis 5 (HYP-FStoTWT): in this scenario, it was decided to change the pattern of admission day. We found in actual data analysis that patients in priority groups tended to stay longer when admitted on Friday or Saturday. We then assumed that as 30\% 
TABLE 1: Admission number (mean \pm SD) and median length of stay (LOS, days) by day as a function of study group.

\begin{tabular}{|c|c|c|c|c|c|c|c|}
\hline & Monday & Tuesday & Wednesday & Thursday & Friday & Saturday & Sunday \\
\hline \multicolumn{8}{|l|}{ Priority group 1} \\
\hline Admission number & $5.8 \pm 2.2$ & $4.6 \pm 2.2$ & $4.7 \pm 2.6$ & $4.4 \pm 2.3$ & $3.9 \pm 2.1$ & $0.8 \pm 1$ & $0.9 \pm 1$ \\
\hline LOS & 9.3 & 10.7 & 9.4 & 10.8 & 14.4 & 14.9 & 10.5 \\
\hline \multicolumn{8}{|l|}{ Priority group 2} \\
\hline Admission number & $33.8 \pm 6.9$ & $31.7 \pm 6.9$ & $32.2 \pm 5.3$ & $26.7 \pm 6$ & $25.4 \pm 5.3$ & $12.8 \pm 3.5$ & $13.7 \pm 3.9$ \\
\hline LOS & 7.1 & 6.9 & 7.0 & 7.7 & 8.3 & 7.1 & 5.4 \\
\hline \multicolumn{8}{|l|}{ Nonpriority group 1} \\
\hline Admission number & $8.6 \pm 2.7$ & $7.9 \pm 2.1$ & $8.3 \pm 2.3$ & $7.8 \pm 2.5$ & $9.3 \pm 3.4$ & $7.3 \pm 2.1$ & $6.2 \pm 2.4$ \\
\hline LOS & 12.9 & 12.0 & 12.3 & 11.9 & 13.0 & 11.8 & 11.5 \\
\hline \multicolumn{8}{|l|}{ Nonpriority group 2} \\
\hline Admission number & $8.7 \pm 3$ & $7.8 \pm 2.7$ & $9.8 \pm 3.6$ & $9.4 \pm 3$ & $8.5 \pm 3.3$ & $7 \pm 2.9$ & $6.5 \pm 2.3$ \\
\hline LOS & 9.7 & 10.2 & 9.5 & 10.5 & 9.7 & 8.7 & 9.5 \\
\hline
\end{tabular}

of these admissions were planned it was possible to modify the admission day. Thirty percent of admitted patients on Friday and Saturday were virtually admitted on Tuesday to Thursday (10\% each day).

(vi) Hypothesis 6 (HYP-PLAN-20\%) reduced the number of planned admissions of PG 2 by $20 \%$ (HYP-PLAN$20 \%$ ), assuming that they can be accommodated by other means (day-care, out-patient consultations).

\section{Results}

3.1. Characteristics of the Actual Series. The actual series included 18,791 hospitalizations; 10,546 (56.1\%) were elective and 6578 (35\%) were through ED with 1667 (8.9\%) transferred patients (413 directly from the ED and 1254 from the ED-OU). Among them, 953 (57.2\%) were aged $\geq 75$ years. Hospitalizations of older patients represented $42 \%$ of through-ED admitted patients, while for elective hospitalizations they represented only $12.5 \%$. Hospitalizations through EDs represented $40 \%$ of hospitalization days. Observed LOS was different as a function of admission mode (elective versus through-ED) $12.2 \pm 3.6$ versus $11.4 \pm 3.8$ days $(P<0.01)$ and age ( $<75$ versus $\geq 75$ years $) 9.7 \pm 0.6$ versus $12.2 \pm 0.6(P<0.01)$.

Table 1 presents the mean number \pm SD of daily hospitalized patients and median hospital-LOS as a function of hospitalization day and PG. A significant reduction was found on weekend days for both elective groups whereas through-ED hospitalizations remained stable. Significant differences were found between study groups for hospital-LOS regarding the day of hospitalization. For elective or planned admissions, Monday hospitalizations were associated with shorter hospital-LOS whereas weekend hospitalizations had significantly longer LOS.

3.2. Validation of the Study Model. Validation of our model and macros consisted of computing actual series admissions in our 354-bed hospital. For this first phase, when stabilized, the mean number of patients boarding in the ED was $24 \pm 5$. In this configuration, and when the system was stabilized, there was a mean of $46 \pm 8$ beds available for all admissions each

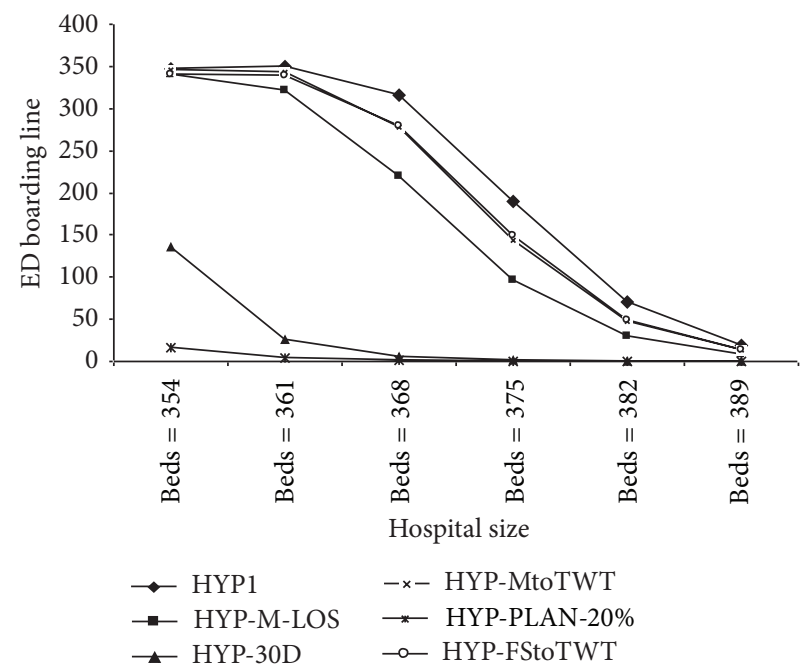

FIGURE 2: Comparison of ED boarding line and hospital size according to different simulations.

morning ( $\min 31, \max 59)$. When patients finally transferred to other institutions were added, the system could never absorb boarders and had an infinite growth.

3.3. Models for Study Hypothesis. The results of these simulations are summarized in Figure 2.

The base hypothesis (HYP1) led to a need for 410 beds $(+16 \%)$ for a stabilized system with a mean ED boarding of $0.5 \pm 0.4 \mathrm{ppn}$. Being more flexible, a hospital of $389(+10 \%)$ beds could manage an acceptable queue of $18.9 \pm 5.5 \mathrm{ppn}$.

In scenarios based on LOS, we found for HYP-M-LOS an ED queue of $29.3 \pm 8.5$ ppn for a 382-bed hospital and $8.3 \pm$ 4.7 ppn for a 389-bed hospital. Limiting the LOS probability distribution at 30 days, HYP-30D, resulted in an average queue of $22.2 \pm 6.6 \mathrm{ppn}$ for only seven beds added to the actual hospital total (361 versus 354 beds). This represents a reduction in LOS for $4 \%$ and $5.4 \%$ of older patients (GP or non-GP, resp.) and $2.6 \%$ and $4.4 \%$ for younger patients. 
An average of 48.1 beds ( 44.7 for 354 beds) was available in the morning.

On scenarios based on pattern and number of admissions, we found that average ED boarding was $14.1 \pm 7.1 \mathrm{ppn}$ with HYP-FStoTWT in a 389-bed hospital. In the morning, 51.7 beds were available for new patients. The effect was more significant when the hospital was smaller. For a 382-bed hospital, average ED boarding was $48.1 \pm 10.5$ ppn. For HYPMtoTWT, in a 382-bed hospital, the boarding queue was an average of $47.4 \pm 7.42$ ppn while for 389 beds the queue was $13 \pm 4.2$ ppn

In scenario HYP-PLAN-20\%, ED boarding in a 354-bed hospital was $16 \pm 4.4 \mathrm{ppn}$ with $45.8 \pm 7.9$ free beds after hospital discharge.

\section{Discussion}

Our results indicate that the ED represents $36 \%$ of overall admissions and up to $40 \%$ of overall hospitalization days to medical wards and the principal hospitalization pathway for patients aged $\geq 75$ years. Daily number of hospitalizations varies according to the day of the week, with a significant reduction for elective groups on weekend days indicating a mismatch between available beds and needs for unplanned hospitalizations. Our results also indicate that hospital-LOS varies as a function of age, hospitalization pathway, and admission day. We found that through-ED hospitalizations were longer by an average of 1 day than elective hospitalizations and elderly patients have a 2-day longer hospital-LOS. Friday and Saturday admissions led to a shorter hospitalLOS when admitted through ED, and elective admissions on Thursday and Friday were 2 days longer. Monday hospitalizations were shorter in the elective admissions group. This phenomenon has also been observed in other facilities [17]. As in other structures [18], besides reduced access to medical services, there is policy of nonadmission to rehabilitation centres or nursing care facilities postponing discharge on Mondays or reluctance to discharge at the weekend [19].

Secondary transfers are the means used to avoid ED overcrowding and access block. Few data are available about transfers from ED-tertiary facilities. However, it has been reported that $1.9 \%$ of ED visits will be transferred [11] and that older patients are at increased risk of transfer [20]. Transfer rate from EDs in Paris area may be $1-2.5 \%$ of all ED visits and around $8-25 \%$ of patients are admitted through EDs [21]. In the present study, the transfer rate was $25.3 \%$ of admitted medical patients through EDs. Furthermore, we found that older patients were at increased risk of transfer to another facility, $46 \%$ were transferred (versus $26 \%$ of younger patients), and they represented $75 \%$ of transferred patients overall. It is currently accepted that transfers are associated with a loss of quality of care, excess costs [12, 22, 23], and a loss of revenue. Reducing transfers from the ED should be a priority.

In this study, we developed simple and easily reproducible sets of simulations, using a validated discrete-event simulation and queuing model [24]. In the first validation model excluding transfers, we found that the mean number of boarding patients $(24 \pm 5)$ was compatible with the ED-OU bed capacity ( 22 beds) and that there was a mean of 46 beds available for all admissions each morning. However, when adding patients finally transferred to other institutions in a second validation test, it was observed that the system can never absorb ED boarding and has an infinite growth, indicating that even if a hospitalization capacity exists, this does not allow all of the patients to be absorbed. Our scenario then included three approaches: an increase of hospital beds, a reduction of LOS, and a reduction in preventable hospitalizations.

In our first hypothesis, we assumed that the clinical context of $5 \%$ of patients $\geq 75$ years and $15 \%$ of other patients transferred from the ED imposed a transfer to another establishment. This hypothesis resulted in a queue of patients boarding in the ED which was incompatible with ED functioning. The increase in hospital beds did not allow reaching acceptable values for ED boarding, except for a hospital of 389 beds. We then tested the impact of a reduction of hospitalLOS. All elective admissions had a LOS for admission on Monday (HYP-M-LOS) (i.e., a reduction of LOS of around $20 \%)$. This model requires an increase in number of medical beds from 354 to $389(+10 \%)$ to achieve a number of ED boarders compatible with the functioning of the ED. In contrast, the model based on a limitation of hospital-LOS to 30 days (HYP-30D) guarantees the on-site admission of transferred patients with an acceptable number of ED boarders associated with a modest increase in beds from 354 to $361(+2 \%)$. This procedure of limiting LOS only concerns a few patients per year (5\% and 3\% of through-ED and elective hospitalizations), which demonstrates its feasibility. Of all the models based on reducing hospital-LOS, only the model limiting hospital-LOS to 30 days appears to be effective and viable.

Using models evaluating the impact of deferring priority admissions for patients $<75$ years old, the hypothesis of admission rescheduling from Monday to another day (HYPMtoTWT) demonstrated that it is impossible to achieve a correct level of ED boarders without an increase in number of beds from 354 to 389 ( $+10 \%)$. In the model of rescheduling admissions planned for Friday or Saturday to another day (HYP-FStoTWT), even with a reduction in LOS, we could only achieve an acceptable number of boarders by increasing the number of beds from 354 to $389(+10 \%)$. These models based on the rescheduling of elective admissions did not enable us to keep all ED patients in our hospital without a $10 \%$ increase in number of hospital beds.

Finally, we tested the possibility of reducing the number of admissions. We tested the hypothesis of a reorientation of $20 \%$ of planned priority patients from group 2 ( $<75$ years) towards other care structures inside the hospital (day-clinics, out-patient consultations) (HYP-PLAN-20\%). This model takes in all ED patients while maintaining an acceptable level of ED boarding without having to increase the number of beds. The occupation level computed for free beds in the morning was coherent with previously published studies [25].

For each of our hypotheses, we tested the impact of increasing the number of medical beds in the hospital from 354 to $389(+10 \%)$. An increase in number of fully staffed inpatient beds is a major determinant of access block and ED 
overcrowding [26]. Clearly, only a limitation of LOS to 30 days or a $20 \%$ reduction in scheduled hospitalizations in persons aged $<75$ years resulted in a compatible number of ED patients.

4.1. Study Limitations. This study has several limitations. First, the program was developed using data originating from a single centre with a local admissions policy. This local admission policy affects more medical units than surgical one but we think that our model can be adapted easily to other systems with only minor modifications. The main criticism is the use of a daily count of admissions versus a real-time count as in other publications [27]. Admissions are computed on a 1-day basis and not an hourly basis. Another limitation of these data is the elective character of direct admissions to hospital. Currently, even if normally entered in the system, it is impossible to differentiate between planned and urgent admissions when the patient is admitted directly to a medical ward. Difficulties also occur in the count of beds. Surprisingly, the total number of beds in hospital is not constant as some beds could be inaccessible due to repairs, sanitary reasons, or a lack of nurses. An exact measure of these closures is not easy due to the random nature of these events and the absence of systematic inventories.

\section{Conclusions}

In conclusion, discrete-event simulation appears to be a powerful tool to evaluate transfer avoidance, the unused admission capacity of the hospital, and the need for additional beds. The simplicity of our macros makes our model very flexible and usable by ED physicians or administrators, easily making assumptions on each computed variable or priorities. Groups are only defined by the order of admission.

Limiting the duration of hospitalization to 30 days in an acute care hospital or reorientation $20 \%$ of scheduled patients $<75$ years old to out-patient care appeared to achieve objectives without increasing the number of beds. A reduction of scheduled patients is not desirable because it decreases hospital resources. The problem of bed blockers is often not a medical problem but usually a social issue. The cost of reducing the LOS of these bed blockers should be assessed against the cost of ED overcrowding and lost admissions. Our model indicates a possible increase in admission capacity. In the context of increasing ED visits and reduction of beds, we suggest that cooperation between medical ward and ED is imperative.

\section{Conflict of Interests}

The authors declare that there is no conflict of interests regarding the publication of this paper.

\section{Acknowledgment}

This research was made in memory of Philippe Hoang Thé Dan.

\section{References}

[1] R. Forero, S. McCarthy, and K. Hillman, "Access block and emergency department overcrowding," Critical Care, vol. 15, no. 2, article 216, 2011.

[2] J. C. Moskop, D. P. Sklar, J. M. Geiderman, R. M. Schears, and K. J. Bookman, "Emergency department crowding, part 1concept, causes, and moral consequences," Annals of Emergency Medicine, vol. 53, no. 5, pp. 605-611, 2009.

[3] S. M. Schneider and B. R. Asplin, "Form follows finance: emergency department admissions and hospital operating margins," Academic Emergency Medicine, vol. 15, no. 10, pp. 959-960, 2008.

[4] R. Biber, H. J. Bail, C. Sieber, P. Weis, M. Christ, and K. Singler, "Correlation between age, emergency department length of stay and hospital admission rate in emergency department patients aged $\geq 70$ years," Gerontology, vol. 59, no. 1, pp. 17-22, 2012.

[5] R. K. Khare, E. S. Powell, G. Reinhardt, and M. Lucenti, "Adding more beds to the emergency department or reducing admitted patient boarding times: which has a more significant influence on emergency department congestion?" Annals of Emergency Medicine, vol. 53, no. 5, pp. 575.e2-585.e2, 2009.

[6] A. J. Forster, I. Stiell, G. Wells, A. J. Lee, and C. Van Walraven, "The effect of hospital occupancy on emergency department length of stay and patient disposition," Academic Emergency Medicine, vol. 10, no. 2, pp. 127-133, 2003.

[7] M. Wargon, B. Guidet, T. D. Hoang, and G. Hejblum, "A systematic review of models for forecasting the number of emergency department visits," Emergency Medicine Journal, vol. 26, no. 6, pp. 395-399, 2009.

[8] D. DeLia, "Annual bed statistics give a misleading picture of hospital surge capacity," Annals of Emergency Medicine, vol. 48, no. 4, pp. 384.e1-388.e2, 2006.

[9] M. J. Vermeulen, J. G. Ray, C. Bell, B. Cayen, T. A. Stukel, and M. J. Schull, "Disequilibrium between admitted and discharged hospitalized patients affects emergency department length of stay," Annals of Emergency Medicine, vol. 54, no. 6, pp. 794-804, 2009.

[10] M. Wargon, E. Casalino, and B. Guidet, "From model to forecasting: a multicenter study in emergency departments," Academic Emergency Medicine, vol. 17, no. 9, pp. 970-978, 2010.

[11] R. Niska, F. Bhuiya, and J. Xu, "National hospital ambulatory medical care survey: 2007 emergency department summary," National Health Statistics Reports, no. 26, pp. 1-31, 2010.

[12] T. Falvo, L. Grove, R. Stachura et al., "The opportunity loss of boarding admitted patients in the emergency department," Academic Emergency Medicine, vol. 14, no. 4, pp. 332-337, 2007.

[13] I. C. Rokos, N. D. Sanddal, A. M. Pancioli, C. Wolff, and D. F. Gaieski, "Inter-hospital communications and transport: turning one-way funnels into two-way networks," Academic Emergency Medicine, vol. 17, no. 12, pp. 1279-1285, 2010.

[14] D. R. Eitel, S. E. Rudkin, M. A. Malvehy, J. P. Killeen, and J. M. Pines, "Improving service quality by understanding emergency department flow: a white paper and position statement prepared for the American academy of emergency medicine," The Journal of Emergency Medicine, vol. 38, no. 1, pp. 70-79, 2010.

[15] A. Bagust, M. Place, and J. W. Posnett, "Dynamics of bed use in accommodating emergency admissions: stochastic simulation model," British Medical Journal, vol. 318, no. 7203, pp. 155-158, 1999. 
[16] M. A. Ross, S. Compton, D. Richardson, R. Jones, T. Nittis, and A. Wilson, "The use and effectiveness of an emergency department observation unit for elderly patients," Annals of Emergency Medicine, vol. 41, no. 5, pp. 668-677, 2003.

[17] A. Earnest, M. I. C. Chen, and E. Seow, "Exploring if day and time of admission is associated with average length of stay among inpatients from a tertiary hospital in Singapore: an analytic study based on routine admission data," BMC Health Services Research, vol. 6, article 6, 2006.

[18] M. R. Carey, H. Sheth, and R. S. Braithwaite, "A prospective study of reasons for prolonged hospitalizations on a general medicine teaching service," Journal of General Internal Medicine, vol. 20, no. 2, pp. 108-115, 2005.

[19] A. M. Varnava, J. E. C. Sedgwick, A. Deaner, K. Ranjadayalan, and A. D. Timmis, "Restricted weekend service inappropriately delays discharge after acute myocardial infarction," Heart, vol. 87, no. 3, pp. 216-219, 2002.

[20] B. Williams, P. Whatmough, and J. Pearson, "Emergency transfer from independent hospitals to NHS hospitals: risk, reasons and cost," Journal of Public Health Medicine, vol. 23, no. 4, pp. 301-305, 2001.

[21] Reseau Cyber urgences: APHP-ARHIF, http://cyber-urgences .aphp.fr/esante/jspSurveillance/statistiqueEpidemioHisto.do.

[22] E. A. Bosk, T. Veinot, and T. J. Iwashyna, "Which patients and where: a qualitative study of patient transfers from community hospitals," Medical Care, vol. 49, no. 6, pp. 592-598, 2011.

[23] B. R. Holroyd, M. J. Bullard, K. Latoszek et al., "Impact of a triage liaison physician on emergency department overcrowding and throughput: a randomized controlled trial," Academic Emergency Medicine, vol. 14, no. 8, pp. 702-708, 2007.

[24] J. L. Wiler, R. T. Griffey, and T. Olsen, "Review of modeling approaches for emergency department patient flow and crowding research," Academic Emergency Medicine, vol. 18, no. 12, pp. 1371-1379, 2011.

[25] M. W. Cooke, S. Wilson, J. Halsall, and A. Roalfe, "Total time in English accident and emergency departments is related to bed occupancy," Emergency Medicine Journal, vol. 21, no. 5, pp. 575$576,2004$.

[26] A. Harris and A. Sharma, "Access block and overcrowding in emergency departments: an empirical analysis," Emergency Medicine Journal, vol. 27, no. 7, pp. 508-511, 2010.

[27] D. C. Lane, C. Monefeldt, and J. V. Rosenhead, "Looking in the wrong place for healthcare improvements: a system dynamics study of an accident and emergency department," Journal of the Operational Research Society, vol. 51, no. 5, pp. 518-531, 2000. 


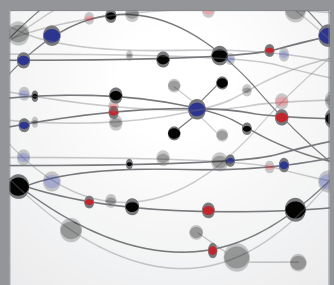

The Scientific World Journal
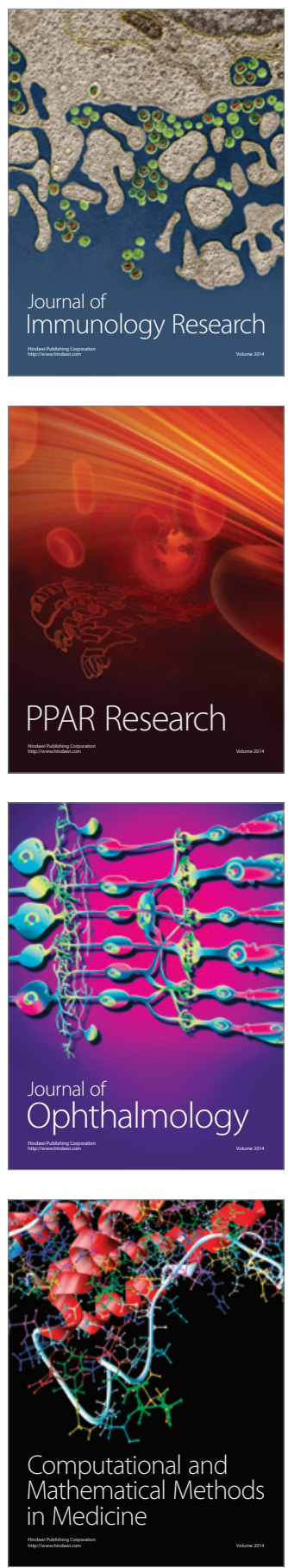

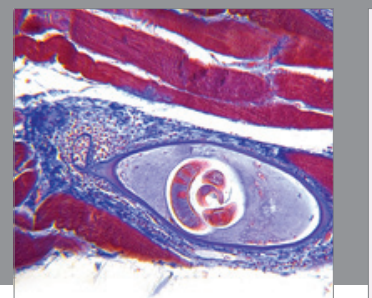

Gastroenterology

Research and Practice
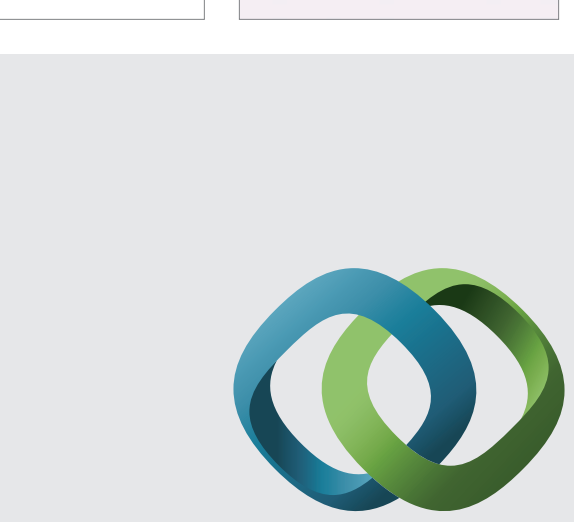

\section{Hindawi}

Submit your manuscripts at

http://www.hindawi.com
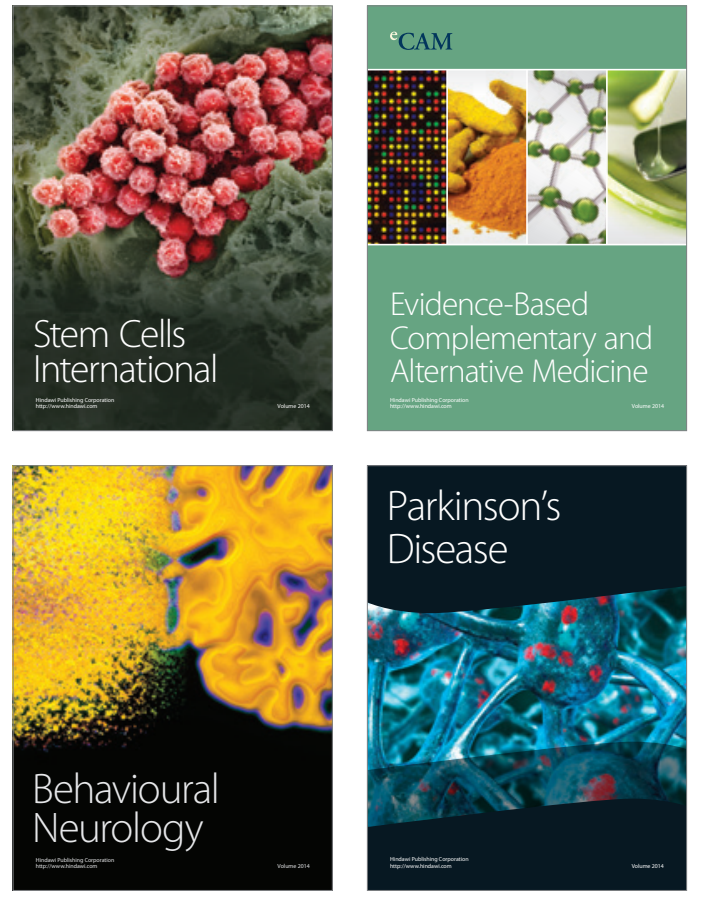
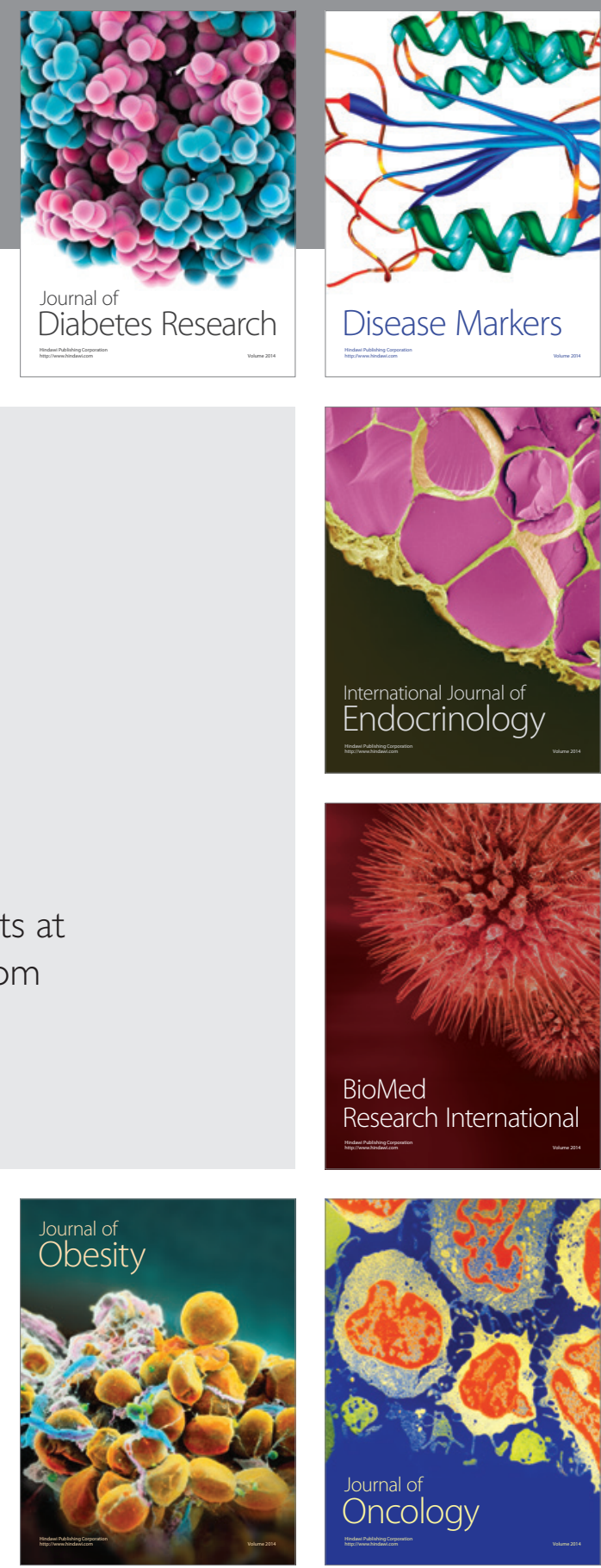

Disease Markers
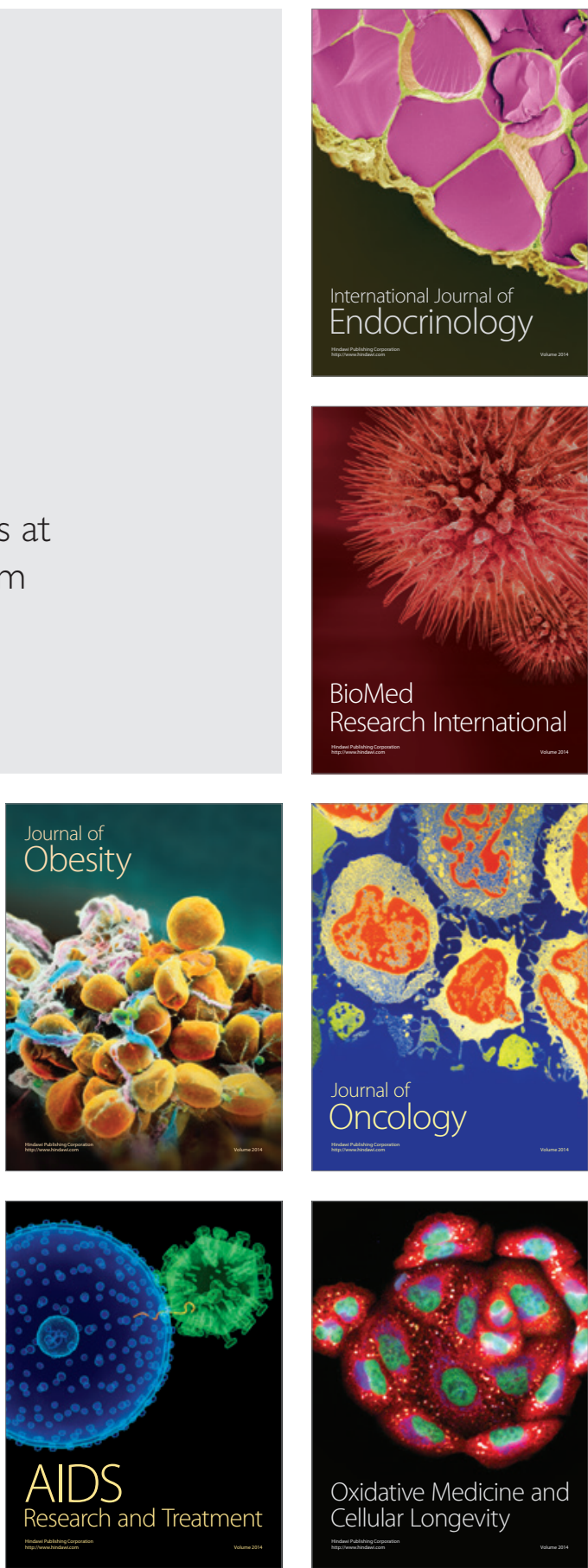